\title{
Exploiting the User-Level Interference Based on Network Coding in D2D Underlaid Cellular Networks
}

\author{
Yijun Guo, ${ }^{1}$ Jing Gao, ${ }^{2}$ and Jianjun Hao ${ }^{1}$ \\ ${ }^{1}$ Beijing Key Laboratory of Network System Architecture and Convergence, Beijing University of Posts and Telecommunications, \\ Beijing 100876, China \\ ${ }^{2}$ School of Electronics and Communication Engineering, Tianjin Normal University, No. 393, Bin Shui West Road, \\ Xiqing District, Tianjin 300387, China
}

Correspondence should be addressed to Yijun Guo; guo.yijun@hotmail.com

Received 29 May 2015; Accepted 25 June 2015

Academic Editor: Qilian Liang

Copyright (C) 2015 Yijun Guo et al. This is an open access article distributed under the Creative Commons Attribution License, which permits unrestricted use, distribution, and reproduction in any medium, provided the original work is properly cited.

Interference is an important and challenging problem faced by the D2D underlaid cellular networks. In this paper, we focus on the user-level interference under multicasting scenarios. Rather than the traditional pairwise way of D2D communications, we propose to implement the information exchanging through a groupwise way. Through introducing the idea of network coding, the proposed scheme is able to utilize the interference as valid signals to enhance the receiving performance, instead of only managing or controlling it. Both theoretical analysis and simulation results prove that the proposed scheme achieves better SINR performance and lower resources occupation than the traditional pairwise D2D transmission schemes.

\section{Introduction}

Device-to-device (D2D) underlaid cellular networks [1] have attracted increasing interests in recent years with the proliferation of wireless devices and the fast progress of mobile computing and wireless network services. It enables nearby pieces of user equipment (UE) to exchange data directly with each other over D2D links without the help of cellular base stations (BS). The advantages of D2D communications lie in high-rate local data transmission, high spectral frequency efficiency by reusing cellular resources, and the capability of lightening the load at BS [2,3]. From user's aspects, the $\mathrm{D} 2 \mathrm{D}$ techniques mainly provides authenticated peer-topeer communications, public safety services, and contextaware services. From operator's aspects, it lies in enhancing the network operation through user cooperation on the D2D links [4]. Recently, 3GPP LTE Rel-12 has a dedicated study item for D2D in which technical specifications are currently being discussed by the industry [5]. D2D underlaid cellular networks became a popular subject for research in recent years, and various studies have been made to increase the performance of the networks and support more advanced mobile services and applications [6-9].

Interference is a severe problem faced by $\mathrm{D} 2 \mathrm{D}$ underlaid cellular networks. On the one hand, since the D2D links reuse the cellular time-frequency resources, the interchannel interference (ICI) between the cellular and D2D links, referred to as cell-level ICI, emerges. On the other hand, the ICI between D2D links themselves, referred to as user-level ICI, increases as the number of D2D users increases since they share limited time-frequency resources. Both cell-level ICI and user-level ICI severely deteriorate the entire system performance [3]. Many efforts have been made in recent years to manage the interference in D2D underlaid cellular networks, in order to improve the spectrum efficiency and ultimately improve the network capacity. In view of spectrum scarcity (a deficit of about $275 \mathrm{MHz}$ by 2014 according to FCC), it is clear that any improvement on the interference control and management side is more than just favorable and eventually shall bring us closer to the yet unknown capacity of cellular networks.

Schemes based on orthogonal resource allocation are studied as direct ways for D2D interference management in 
[10-14]. Works in [10-12] mainly focus on the cell-level ICI. The principle is to assign orthogonal time frequency to D2D user equipment (DUE) and cellular user equipment (CUE). Authors in $[13,14]$ introduce specialized schemes for userlevel interference such as maximum carrier-to-interference ratio (MCI) scheduling and maximum throughput (MT) scheduling. However, these orthogonal schemes have not considered the spectrum efficiency.

For efficiency enhancement, nonorthogonal frequency reuse strategies are investigated in $[3,15-17]$. $\delta D$-interference limited area control scheme is proposed to manage interference in [3]. It limits DUE's maximum transmit power and excludes the CUEs from the $\delta D$-interference limited area, in order to minimize the interference to DUEs. In [15], three receiving modes are switched at the D2D receiver according to the interference level in order to improve the D2D performance in terms of outage probability. The mind of cooperative interference cancellation (CIC) has been introduced in [16]. CIC exploits the interference correlation between closeby users to eliminate interference cooperatively and improve downlink throughput. The authors in [17] adopt interference alignment (IA) to mitigate the cell-level interference and improve capacity. This paper intends to adopt interference alignment (IA) for improving the energy efficiency (EE) of both the device-to-device (D2D) and cellular links in a D2D multiple-input and multiple-output (MIMO) downlink underlaying cellular network. For this case, a critical issue is to properly coordinate existing interference to guarantee both reliable macro cellular and D2D communications as they simultaneously reuse the same frequency and time resources. However, these schemes only treat the interference as a negative factor and focus on controlling and eliminating but do not consider utilizing the interference for better receiving.

In D2D underlaid cellular networks, it is important and challenging to manage the interference in the presence of limited resources and large amount of users. To deal with the interference problem and improve network capacity discussed above, in this work, we focus on the user-level interference under multicasting scenarios and propose to introduce the idea of network coding (NC) into D2D underlaid cellular networks. Rather than the traditional pairwise way, we propose to implement the information exchanging process through a groupwise way and utilize the interference to enhance the receiving performance. Besides serving as a physical transmission scheme for multicasting services in cellular networks, we expect the proposed scheme to be applicable in other network research fields for a higher network performance.

The remainder of this paper is organized as follows. Section 2 gives the system model; Section 3 describes the proposed NC-based groupwise (NC-G) transmission scheme; Section 4 analyzes the theory performance; Section 5 simulates and compares the NC-G scheme with another two traditional schemes; Section 6 concludes the paper.

Notations. Lowercase boldface letters represent vectors. $|\cdot|$ returns the absolute value of a scalar and $\|\cdot\|$ gives the norm of a vector.

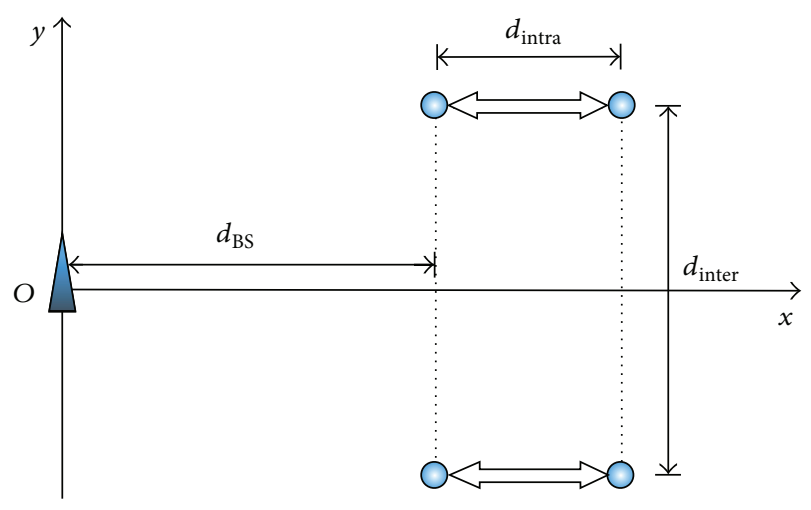

Figure 1: System model.

\section{Background and Foundation}

2.1. System Model. Consider D2D underlaid cellular networks consisting of one BS and 2 D2D pairs. Use a two-dimensional plane to represent their positions. Place the BS node at the original point and place the 4 DUE nodes as a rectangle as shown in Figure 1. The topology is determined by three kinds of distance: $d_{\text {intra }}$ denotes the distance between two DUEs in a pair; $d_{\text {inter }}$ denotes the distance between the centers of two pairs; $d_{\mathrm{BS}}$ denotes the distance between BS and the rectangular center.

D2D communications are established and share timefrequency resources with the cellular communications through central controlling from BS using dedicated control channels. Use physical resource block (PRB) to measure the time-frequency resources. The transmitting and receiving of each DUE node are implemented with different PRBs.

The base station (BS) need to broadcast a block of data to the $4 \mathrm{D} 2 \mathrm{D}$ user equipments (DUEs). To alleviate its own load, BS distributes the block of data to each DUE and initiates D2D communications to exchange information locally. Each DUE, denoted by $D_{i}(i \in[1,4])$, has been allocated data of length $N$ to exchange, denoted by $\mathbf{x}_{i}=\left[x_{i, 1}, \ldots, x_{i, N}\right]$. As for D2D communication, assume that $M$-ary modulation is adopted for all the DUEs; the D2D links are block fading, and perfect channel state information (CSI) is known at the receivers.

2.2. The Traditional Way. The proposed scheme detailed later will be compared with the traditional underlaid D2D communication scheme based on pairwise transmissions. Based on pairwise way, the 4 DUEs as discussed in Section 2.1 have to establish communications and exchange their information in pairs.

Below we give a simple pairwise transmission scheme. A complete information exchange process between the 4 DUEs contains three stages, as listed in Table 1, where $D_{x} \leftrightarrow$ $D_{y}$ represents the notion that the communication channel between $D_{x}$ and $D_{y}$ is constructed and they could exchange information with each other. In particular, under the communication target described in Section 2.1, in the first stage, $D_{1}$ and $D_{2}$ exchange $\mathbf{x}_{1}$ and $\mathbf{x}_{2}$ while $D_{3}$ and $D_{4}$ exchange $\mathbf{x}_{3}$ and $\mathbf{x}_{4}$. In the following stages, the two pairs are interchanged for 
TABLE 1: Scheduling for exchanging data between 4 DUEs by pairwise D2D communications.

\begin{tabular}{lll}
\hline Phase 1 & $D_{1} \leftrightarrow D_{2}$ & $D_{3} \leftrightarrow D_{4}$ \\
Phase 2 & $D_{1} \leftrightarrow D_{3}$ & $D_{2} \leftrightarrow D_{4}$ \\
Phase 3 & $D_{1} \leftrightarrow D_{4}$ & $D_{2} \leftrightarrow D_{3}$ \\
\hline
\end{tabular}

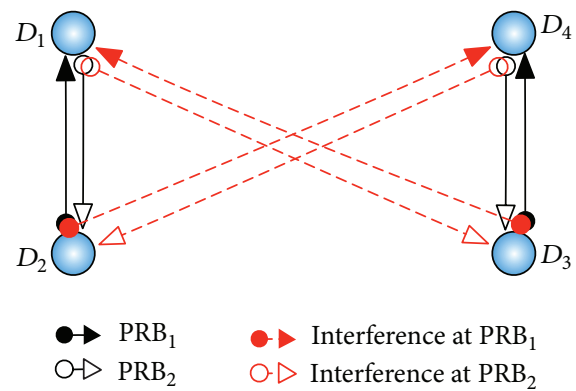

FIGURE 2: Interference model under traditional pairwise D2D transmission.

similar pairwise information exchanging. After three stages, each DUE node has exchanged information with 3 other DUE nodes. Thus, all of the 4 DUEs obtain the complete data $\mathbf{x}=$ $\left[\mathbf{x}_{1}, \mathbf{x}_{2}, \mathbf{x}_{3}, \mathbf{x}_{4}\right]$.

\section{Problem Description}

As mentioned above, there are two kinds of interferences in an underlaid cellular D2D network: cell-level ICI and userlevel ICI. The former one has been researched deeply and would not be discussed in this paper. When limited PRBs are assigned for D2D communications, the user-level ICI is a severe problem especially when the amount of DUEs is large.

In particular, as for the pairwise D2D way, if two D2D pairs positioned in each other's communication range are assigned by the same PRBs, they would interfere with each other as illustrated in Figure 2. For example, let $\mathscr{M}(\cdot)$ denote the modulation function and let $\mathbf{s}_{i}=\left[s_{i, 1}, \ldots, s_{i, N}\right]$ denote the symbol vector modulated by $\mathbf{x}_{i}$; that is, $\mathbf{s}_{i}=\mathscr{M}\left(\mathbf{x}_{i}\right)$. Assume each symbol $s_{i, n}$ is energy normalized; that is, $E\left[\left\|s_{i, n}\right\|\right]=1$.

Focus on the receiving at $D_{1}$. The valid signal is $\mathbf{s}_{2}$ from $D_{2}$ through $\mathrm{PRB}_{1}$. Since $D_{3}$ also uses $\mathrm{PRB}_{1}$ to transmit $\mathbf{s}_{3}$ to $D_{4}$, the signal received by $D_{1}$ is interfered with and is formulated as

$$
\mathbf{r}_{D_{1}}=\sqrt{\alpha_{21} P_{2}} \mathbf{h}_{21} \mathbf{s}_{2}+\sqrt{\alpha_{31} P_{3}} \mathbf{h}_{31} \mathbf{s}_{3}+\mathbf{n}_{D_{1}},
$$

where $\alpha_{i j}$ characterizes the path loss effect of channel $D_{i} \rightarrow$ $D_{j} . P_{i}$ constrains the transmit power at $D_{i} . \mathbf{h}_{i j}$ denotes the multipath fading item with its entries modeled as independent and identically distributed (i.i.d.) Rayleigh variables. $\mathbf{n}_{D_{i}} \sim \mathscr{C} \mathscr{N}(0,1)$ models the normalized additive Gaussian noise at the receiver of $D_{i}$.

According to (1), the received signal-to-noise-plusinterference ratios (SINR) at $D_{1}$ are given by

$$
\gamma_{1}=\frac{\alpha_{21} P_{2}\left|\mathbf{h}_{21}\right|^{2}}{\alpha_{31} P_{3}\left|\mathbf{h}_{31}\right|^{2}+1} .
$$

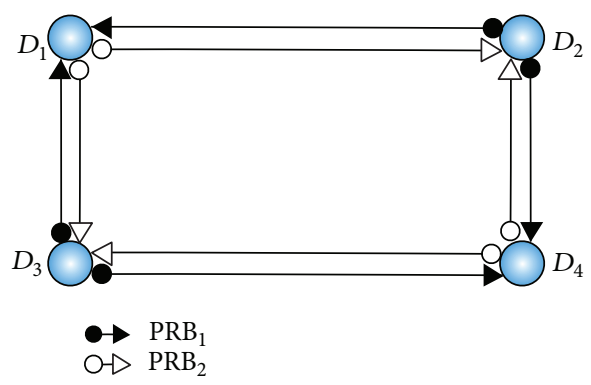

FIGURE 3: The proposed group-based transmission model.

Obviously, the valid signals from $D_{3}$ are interfered with by the signals from $D_{4}$. Besides, given transmit power, the receiving performance would degrade when $\alpha_{41}$ increases; that is, the two D2D pairs become close.

Based on the above analysis, user-level ICI management is an important and challenging problem in underlaid D2D networks, and it is our major concern in this work. In the following sections, we will show a new group-based transmission scheme for multicasting scenarios. Rather than controlling or eliminating the interference by traditional schemes, the proposed scheme is able to exploit the user-level ICI to improve the receiving performance using the idea of network coding.

\section{The Proposed Scheme}

With the proposed NC-based groupwise scheme, two D2D pairs, that is, 4 DUE nodes, are organized as a group to implement the information exchanging process, as illustrated in Figure 3. Assume that all of the 4 DUEs are synchronized through the BS. The synchronization problem in realization would be discussed later. $D_{1}$ and $D_{4}$ use $P_{R B}$ to receive and $\mathrm{PRB}_{2}$ to transmit. $D_{2}$ and $D_{3}$ are just opposite, using $\mathrm{PRB}_{2}$ to receive and $P R B_{1}$ to transmit.

We propose to exploit the idea of network coding in order to enable the simultaneous receiving of two distinct signals and also improve the transmission efficiency. In particular, focus on the exchanging of the $n$th symbols to describe the proposed scheme. A complete exchange of the $n$th symbols is defined as each DUE obtaining the $n$th symbols from all the DUEs, that is, $\left[s_{1, n}, s_{2, n}, s_{3, n}, s_{4, n}\right]$. This process contains two stages, referred to as the precoding (PC) stage and the network coding $(\mathrm{NC})$ stage, respectively.

4.1. The PC Stage. Initially, each of the 4 DUEs only has its own codeword, that is, $x_{1, n}, x_{2, n}, x_{3, n}$, and $x_{4, n}$, respectively. The PC stage occupies two time slots (TS). At the transmitter of each DUE node, for example, $D_{i}$, it precodes its own symbol $s_{i, n}$ by different coefficients in the two time slots, denoted by $c_{i}^{(1)}$ and $c_{i}^{(2)}$, respectively, and then sends them to its two neighbor receivers. At the receiver, each DUE receives superposition of two signals from the two neighbors in each TS.

Focus on the reception at $D_{1}$ for illustration. $D_{1}$ receives signals from both $D_{2}$ and $D_{3}$ simultaneously. For the assumption of block fading, the channel fading coefficients could be 
Table 2: Precoding coefficients in the PC stage.

\begin{tabular}{lcccc}
\hline$c_{i}^{(j)}$ & $D_{1}$ & $D_{2}$ & $D_{3}$ & $D_{4}$ \\
\hline $\mathrm{TS}^{(1)}$ & 1 & 1 & 1 & 1 \\
$\mathrm{TS}^{(2)}$ & 1 & 1 & -1 & -1 \\
\hline
\end{tabular}

seen as the same during the whole exchanging process of the $n$th symbols. Thus, the superposition signals received by $D_{1}$ during $\mathrm{TS}^{(1)}$ and $\mathrm{TS}^{(2)}$, respectively, are formulated as

$$
\begin{aligned}
r_{D_{1}, n}^{(1)}= & \sqrt{\alpha_{21} P_{2}} h_{21, n} c_{2}^{(1)} s_{2, n}+\sqrt{\alpha_{31} P_{3}} h_{31, n} c_{3}^{(1)} s_{3, n} \\
& +n_{D_{1}, n}^{(1)}, \\
r_{D_{1}, n}^{(2)}= & \sqrt{\alpha_{21} P_{2}} h_{21, n} c_{2}^{(2)} s_{2, n}-\sqrt{\alpha_{31} P_{3}} h_{31, n} c_{3}^{(2)} s_{3, n} \\
& +n_{D_{1}, n}^{(2)},
\end{aligned}
$$

where $r_{D_{i}, n}^{(j)}(j=1,2)$ represents the reception at $D_{i}$ in $\operatorname{TS}^{(j)}$ for the $n$th symbol. Notice here that the signal from $D_{4}$ is transmitted through $\mathrm{PRB}_{1}$, which would not interfere with the receiving at $D_{1}$ through $\mathrm{PRB}_{2}$.

With the two received signals, $D_{1}$ is able to recover $s_{2, n}$ and $s_{3, n}$ if and only if the two precoding vectors $\left[c_{2}^{(1)}, c_{3}^{(1)}\right]$ and $\left[c_{2}^{(2)}, c_{3}^{(2)}\right]$ are linearly independent. For simplicity, specify the precoding coefficients used by the 4 DUEs in the PC stage as shown in Table 2 . Hence, at $D_{1}, s_{2, n}$ and $s_{3, n}$ can be recovered through simple Gaussian eliminations as

$$
\begin{aligned}
& \hat{s}_{2, n}^{1}=\frac{1}{2 \sqrt{\alpha_{21} P_{2}} h_{21, n}}\left(r_{D_{1}, n}^{(1)}+r_{D_{1}, n}^{(2)}\right), \\
& \hat{s}_{3, n}^{1}=\frac{1}{2 \sqrt{\alpha_{31} P_{3}} h_{31, n}}\left(r_{D_{1}, n}^{(1)}-r_{D_{1}, n}^{(2)}\right),
\end{aligned}
$$

where $\widehat{s}_{i, n}^{1}$ denotes the recovered version of $s_{i, n}$ at $D_{1}$. Then $D_{1}$ can demodulate $\widehat{s}_{2, n}$ and $\widehat{s}_{3, n}$ to get the codewords $x_{2, n}$ and $x_{3, n}$ as

$$
\begin{aligned}
& \hat{x}_{2, n}^{1}=\mathscr{M}^{-1}\left(\widehat{s}_{2, n}^{1}\right), \\
& \hat{x}_{3, n}^{1}=\mathscr{M}^{-1}\left(\hat{s}_{3, n}^{1}\right) .
\end{aligned}
$$

4.2. The NC Stage. Next, the NC stage occupies one TS to complete the whole exchange of $\left[x_{1, n}, x_{2, n}, x_{3, n}, x_{4, n}\right]$ between the 4 DUEs. The transmitter of each DUE node performs linear network coding, for example, XOR function to the two codewords it has already obtained during the previous PC stage. Then it remodulates and sends the encoded codewords through its transmit PRB. At the receiver, each DUE receives superposition of two copies of the encoded symbol from its two neighbors. Through demodulating the superposition signal to get the encoded codeword, and then decoding it using local codeword information, each DUE can obtain the last unknown codeword. So far, the whole exchanging process for the $n$th symbols between all the 4 DUEs is completed.
Focus on the reception at $D_{1}$ for illustration, again. Through the previous PC stage, its two neighbors $D_{2}$ and $D_{3}$ have already gotten the information from $D_{1}$ and $D_{4}$, denoted by $\left[\hat{x}_{1, n}^{2}, \widehat{x}_{4, n}^{2}\right]$ and $\left[\hat{x}_{1, n}^{3}, \widehat{x}_{4, n}^{3}\right]$, respectively. In $\mathrm{TS}^{(3)}, D_{2}$ and $D_{3}$ perform network coding to the recovered codewords and then modulate the network coded codeword as

$$
\begin{aligned}
& s_{1 \oplus 4, n}^{2}=\mathscr{M}\left(\hat{x}_{1, n}^{2} \oplus \hat{x}_{4, n}^{2}\right), \\
& s_{1 \oplus 4, n}^{3}=\mathscr{M}\left(\hat{x}_{1, n}^{3} \oplus \hat{x}_{4, n}^{3}\right),
\end{aligned}
$$

respectively. Correspondingly, $D_{1}$ receives the superposition of these two copies of the network coded symbol $s_{1 \oplus 4, n}$, formulated as

$$
r_{D_{1}, n}^{(3)}=\sqrt{\alpha_{21} P_{2}} h_{21, n} s_{1 \oplus 4, n}^{2}+\sqrt{\alpha_{31} P_{3}} h_{31, n} s_{1 \oplus 4, n}^{3}+n_{D_{1}, n}^{(3)} .
$$

In order to get $x_{4, n}, D_{1}$ first applies the combining receiving method to the superposition signal and detects $s_{1 \oplus 4, n}$ as

$$
\hat{s}_{1 \oplus 4, n}^{1}=\frac{r_{D_{1}, n}^{(3)}}{\sqrt{\alpha_{21} P_{2}} h_{21, n}+\sqrt{\alpha_{31} P_{3}} h_{31, n}} .
$$

Then $D_{1}$ demodulates $\widehat{s}_{1 \oplus 4, n}^{1}$ to get the encoded codeword as

$$
\widehat{x}_{1 \oplus 4, n}^{1}=\mathscr{M}^{-1}\left(\widehat{s}_{1 \oplus 4, n}^{1}\right) \text {. }
$$

Finally, $D_{1}$ decodes $\hat{x}_{1 \oplus 4, n}^{1}$ using its local codeword $x_{1, n}$ to obtain $x_{4, n}$ as

$$
\widehat{x}_{4, n}^{1}=\widehat{x}_{1 \oplus 4, n}^{1} \oplus x_{1, n} .
$$

Through the PC stage and the NC stage, $D_{1}$ gets the whole $n$th symbols $\widehat{\mathbf{x}}_{n}^{1}=\left[\hat{x}_{1, n}^{1}, \hat{x}_{2, n}^{1}, \widehat{x}_{3, n}^{1}, \hat{x}_{4, n}^{1}\right]$. As for $D_{2}, D_{3}$, and $D_{4}$, the communication processes are similar with $D_{1}$. As described above, for each DUE, both signals from the two neighbors transmitting in the same PRB are valid signals rather than interference. Hence the purpose of exploiting interference to improve the receiving performance is realized.

4.3. Realization Problems. Two realization problems would be discussed in this subsection.

4.3.1. Synchronization. Intuitively, the proposed scheme is highly dependent on the synchronization between the 4 DUEs, which seems difficult to realize. However, the stress is relieved in cellular-controlled D2D networks. The reason is that the centralized control mode allows every DUE to be synchronized with the center node, that is, the BS. On the other hand, the synchronization offset caused by the transmission delay between DUE nodes can be negligible since a D2D communication is established only if the DUEs are close.

4.3.2. Assignment of Precoding Coefficients. As described in the scheme, the 4 DUEs need to be preassigned the precoding coefficients for the PC stage. This assignment can be implemented easily by the BS through its control channels with all of the DUEs during the establishment stage of the D2D communication. 


\section{Performance Analysis}

From a statistical point of view, the 4 DUEs are symmetric and equivalent. Without loss of generality, focus on $D_{1}$ to analyze its SINR performance and the results can be extended to the other DUE nodes.

5.1. The SINR of the Proposed Scheme. The proposed scheme has different SINR in its two stages. In the PC stage, the combined received signal for the decoding of $s_{2, n}$ is

$$
r_{D_{1}, n}^{(1)}+r_{D_{1}, n}^{(2)}=2 \sqrt{\alpha_{21} P_{2}} h_{21, n} s_{2, n}+n_{D_{1}, n}^{(1)}+n_{D_{1}, n}^{(2)}
$$

which corresponds to SINR of $2 \alpha_{21} P_{2}\left|h_{21, n}\right|^{2}$. Similarly, the combined received signal for the decoding of $s_{3, n}$ is

$$
r_{D_{1}, n}^{(1)}-r_{D_{1}, n}^{(2)}=2 \sqrt{\alpha_{31} P_{3}} h_{31, n} s_{3, n}+n_{D_{1}, n}^{(1)}-n_{D_{1}, n}^{(2)}
$$

which corresponds to SINR of $2 \alpha_{31} P_{3}\left|h_{31, n}\right|^{2}$. Hence, the average SINR during the PC stage can be calculated as

$$
\bar{\gamma}_{D_{1}, n}^{\mathrm{PC}}=\alpha_{21} P_{2}\left|h_{21, n}\right|^{2}+\alpha_{31} P_{3}\left|h_{31, n}\right|^{2} .
$$

In the NC stage, since $s_{1, n}$ is known by $D_{1}$ locally, the decoding performance of $s_{4, n}$ equals $s_{1 \oplus 4, n}$. View the two versions of $s_{1 \oplus 4, n}$ from $D_{2}$ and $D_{3}$, that is, $s_{1 \oplus 4, n}^{2}$ and $s_{1 \oplus 4, n}^{3}$, as the same information; the combined received signal can be formulated as

$$
r_{D_{1}, n}^{(3)}=\left(\sqrt{\alpha_{21} P_{2}} h_{21, n}+\sqrt{\alpha_{31} P_{3}} h_{31, n}\right) s_{1 \oplus 4, n}+n_{D_{1}, n}^{(3)} .
$$

The corresponding instant SINR which equals the SINR in the NC stage is shown as

$$
\gamma_{D_{1}, n}^{\mathrm{NC}}=\left|\alpha_{21} P_{2} h_{21, n}+\alpha_{31} P_{3} h_{31, n}\right|^{2} .
$$

5.2. Comparison with the Traditional Scheme. Next, we compare the SINR performance of the proposed scheme and the traditional scheme. For the whole data $\mathbf{x}_{i}=\left[x_{i, 1}, \ldots, x_{i, N}\right]$, according to (2), the SINR of the traditional scheme is formulated as

$$
\gamma_{D_{1}}^{T}=\frac{\alpha_{21} P_{2}\left|\mathbf{h}_{21}\right|^{2}}{\alpha_{31} P_{3}\left|\mathbf{h}_{31}\right|^{2}+1} .
$$

According to (13) and (15), the SINRs of the proposed scheme in the two stages are, respectively, written as

$$
\begin{aligned}
& \overline{\boldsymbol{\gamma}}_{D_{1}}^{\mathrm{PC}}=\alpha_{21} P_{2}\left|\mathbf{h}_{21}\right|^{2}+\alpha_{31} P_{3}\left|\mathbf{h}_{31}\right|^{2}, \\
& \boldsymbol{\gamma}_{D_{1}}^{\mathrm{NC}}=\left|\alpha_{21} P_{2} \mathbf{h}_{21}+\alpha_{31} P_{3} \mathbf{h}_{31}\right|^{2} .
\end{aligned}
$$

With a simple deduction, we prove that $\bar{\gamma}_{D_{1}}^{\mathrm{PC}} \geq \boldsymbol{\gamma}_{D_{1}}^{T}$. Besides, $\gamma_{D_{1}}^{\mathrm{NC}} \geq \boldsymbol{\gamma}_{D_{1}}^{T}$ is a big probability event when the distance from $D_{3}$ to $D_{1}$ has a magnitude equal to the distance from $D_{2}$ to $D_{1}$.
TABLE 3: Simulation parameters setting.

\begin{tabular}{lccc}
\hline & Parameters & Values & Unit \\
\hline \multirow{3}{*}{ Topology } & $d_{\mathrm{BS}}$ & 1000 & $\mathrm{~m}$ \\
& $d_{\text {intra }}$ & $5 \sim 20$ & $\mathrm{~m}$ \\
& $d_{\text {inter }}$ & $5 \sim 20$ & $\mathrm{~m}$ \\
\hline \multirow{3}{*}{ Channel modeling } & $n$ & 1.6 & - \\
& $d_{0}$ & 1 & $\mathrm{~m}$ \\
& $K_{\mathrm{dB}}$ & $-40 \sim 15$ & $\mathrm{~dB}$ \\
\hline \multirow{3}{*}{ Transmission } & $f_{c 1}$ & $1.5 \times 10^{9}$ & $\mathrm{~Hz}$ \\
& $f_{c 2}$ & $1.6 \times 10^{9}$ & $\mathrm{~Hz}$ \\
& $P_{t}$ & 1 & $\mathrm{~W}$ \\
& $N_{0}$ & $-70 \sim 20$ & $\mathrm{dBm}$ \\
\hline
\end{tabular}

\section{Simulations}

6.1. Simulation Scenario and Schemes. Three schemes are simulated and compared in this section:

(1) NC-G Scheme: the proposed NC-based groupwise transmission scheme. Use function $\mathscr{F}(\cdot)$ to represent the amount of used physical resources blocks. Thus, it holds that

$$
\mathscr{F}(\mathrm{NC}-\mathrm{G})=1 .
$$

(2) OF-P scheme: the traditional pairwise transmission with orthogonal resources. Each D2D pair occupies a distinct PRB to exchange information; thus we have

$$
\mathscr{F}(\mathrm{OF}-\mathrm{P})=2 \text {. }
$$

(3) IF-P scheme: the traditional pairwise transmission with identical resource. Two pairs use the same PRB for transmission; thus we have

$$
\mathscr{F}(\mathrm{IF}-\mathrm{P})=1 .
$$

6.2. Simulation Parameters Setting. The path loss effect is characterized by the log distance path loss model [18], formulated as

$$
\mathrm{PL}_{\mathrm{dB}}=-20 \log _{10}\left(\frac{\lambda}{4 \pi d_{0}}\right)+10 n \log _{10}\left(\frac{d}{d_{0}}\right),
$$

where $n$ is the path loss index, $d_{0}$ is the reference distance, $\lambda=$ $\left(3 \times 10^{8}\right) / f_{c}$ is the wavelength of the carrier waves, and $\mathrm{PL}_{\mathrm{dB}}$ represents the path loss in the unit of $\mathrm{dB}$. As for the transmission within buildings with line of sight (LOS), $n$ is set in the range of [1.6-1.8], and $d_{0}$ takes value near 1 when the transmission distance is no bigger than 1 kilometer. The multipath fading effect is modeled as the Rice fading with index $K$.

The simulation parameters are set as shown in Table $3.10^{6}$ symbols for each DUE had been exchanged to get stabilized simulation results.

\subsection{Simulation Results}

6.3.1. SINR. The received SINRs are simulated to observe the receiver performance intuitively. 


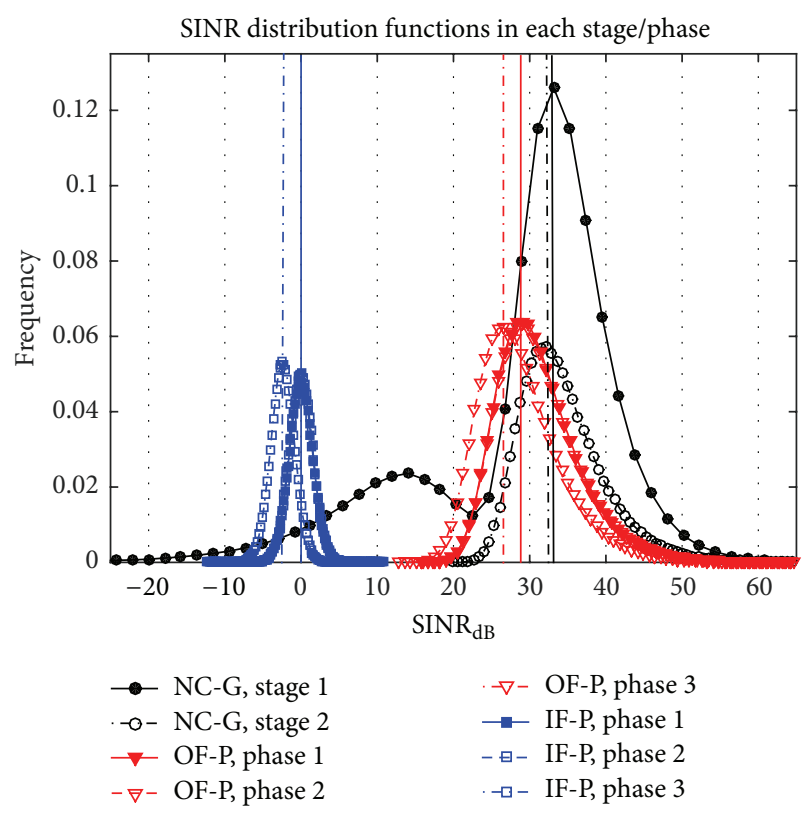

FIGURE 4: SINR distribution functions in each stage/phase of the three schemes.

Figure 4 compares the SINR distribution functions of the three schemes in each stage (NC-G) or each phase (OF-P and IF-P). Define $\mathbf{d}=\left[d_{\text {intra }}, d_{\text {inter }}\right]$ as the topology parameter vector. The parameters are set as $\mathbf{d}=[5,5], K=15$, and $N_{0}=-40$. The result shows that, within the NC-G scheme, although the signals received in stage 1 and stage 2 are distributed differently, the positions of their peaks are nearly the same. Within both the OF-P and the IF-P schemes, the signals received in phase 1 and phase 2 have the same distribution, which is better than phase 3 . This is because, in phase 3, DUEs on the diagonal of the square are paired to communicate. Longer distance causes worse SINR. As for the comparison between three schemes, we observe that NC-G is the best, OF-P is inferior, and IF-P is the worst.

Next, we fix $\mathbf{d}=[5,5]$ and $N_{0}=-40 \mathrm{dBm}$ and vary $K$ from $-40 \mathrm{~dB}$ (Rayleigh distribution) to $15 \mathrm{~dB}$ (Gaussian distribution). Use the SINRs in stage 1 of NC-G and phase 1 of OF-P/IF-P to represent each scheme. The results are illustrated in Figure 5. With different Rice index $K$, the position of the peak value in each scheme only moves a little. However, as $K$ decreases, the SINR curve of each scheme becomes more diffuse, which implies that the variance increases.

Then, we fix $\mathbf{d}=[5,5]$ and $K=0$ and vary $N_{0}$ from $-70 \mathrm{dBm}$ to $70 \mathrm{dBm}$. The results are shown in Figure 6. The increase of $N_{0}$ leads to a leftward shift in the SINR curve of each scheme but hardly changes the shape of each curve.

6.3.2. SER. The average symbol error rate (SER) at receivers is simulated under different channel fading and topology.

When $\mathbf{d}=[5,5]$, the cases where $K=-30 \mathrm{~dB}$, $0 \mathrm{~dB}$, and $15 \mathrm{~dB}$ are simulated. The curves of SER with $N_{0}$ changes are plotted in Figure 7. The result corresponding to $K=15 \mathrm{~dB}$ performs the best since the channel fading obeys an approximate Gaussian distribution. As $K=15 \mathrm{~dB}$

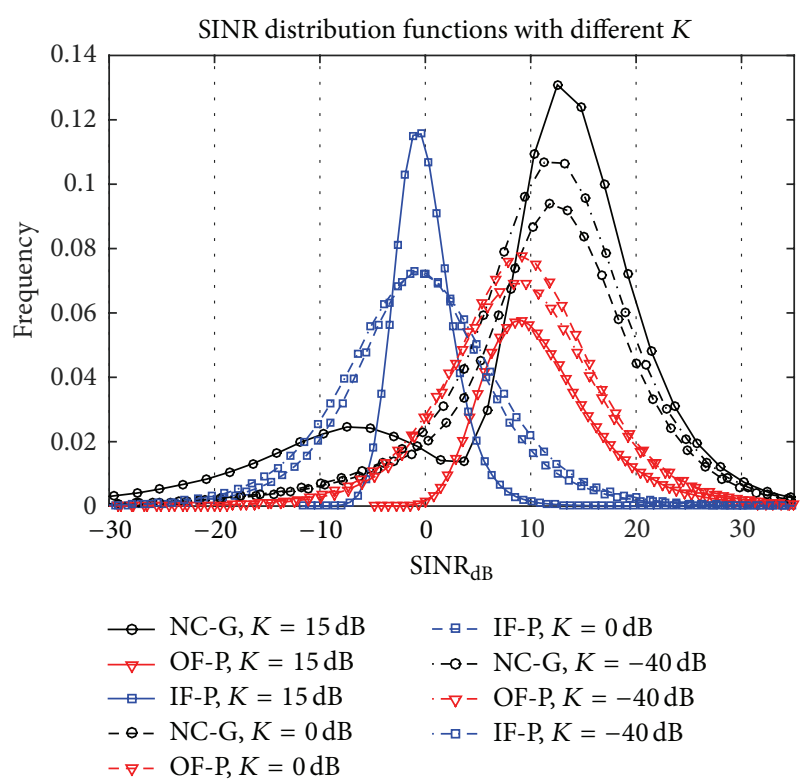

FIGURE 5: SINR distribution functions in stage 1 of the three schemes with different $K$.

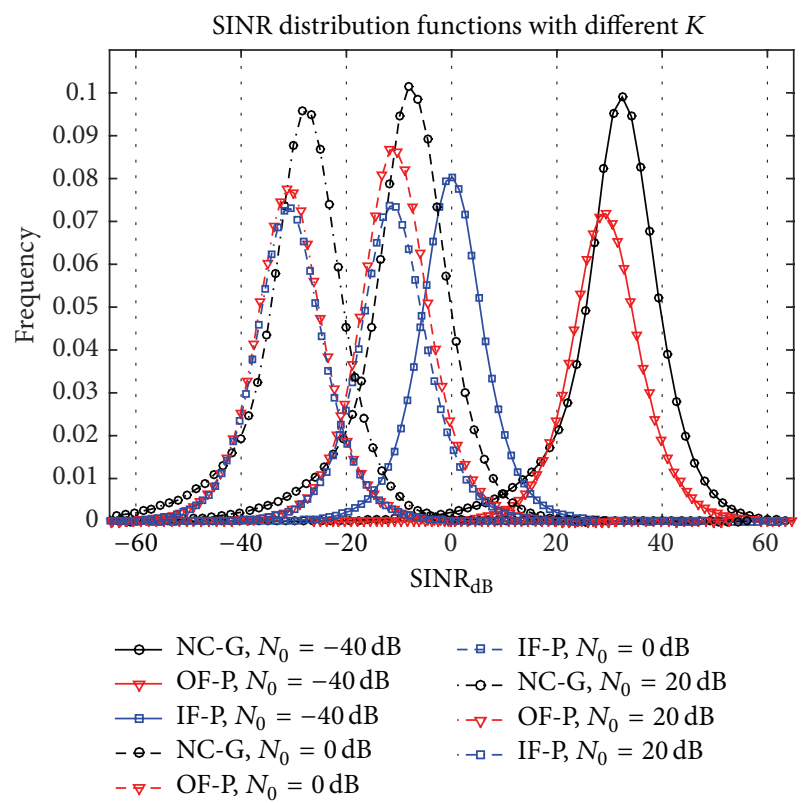

FIGURE 6: SINR distribution functions in stage 1 of the three schemes with different $N_{0}$.

increases, the SER performance degrades severely. Besides, the performance gains brought by the NC-G also degrade. However, the NC-G scheme performs better than the other two schemes in all of these cases.

Finally, we fixed $K=0 \mathrm{~dB}$ and changed $\mathbf{d}$ to observe the SER performance under different topology. The results illustrated in Figure 8 show that when the average of $d_{\text {intra }}$ and $d_{\text {inter }}$ increases, for example, changing $\mathbf{d}$ from $[10,10]$ to $[20,20]$, the SER performance degrades correspondingly. However, when the average distance is fixed and $d_{\text {intra }} / d_{\text {inter }}$ 


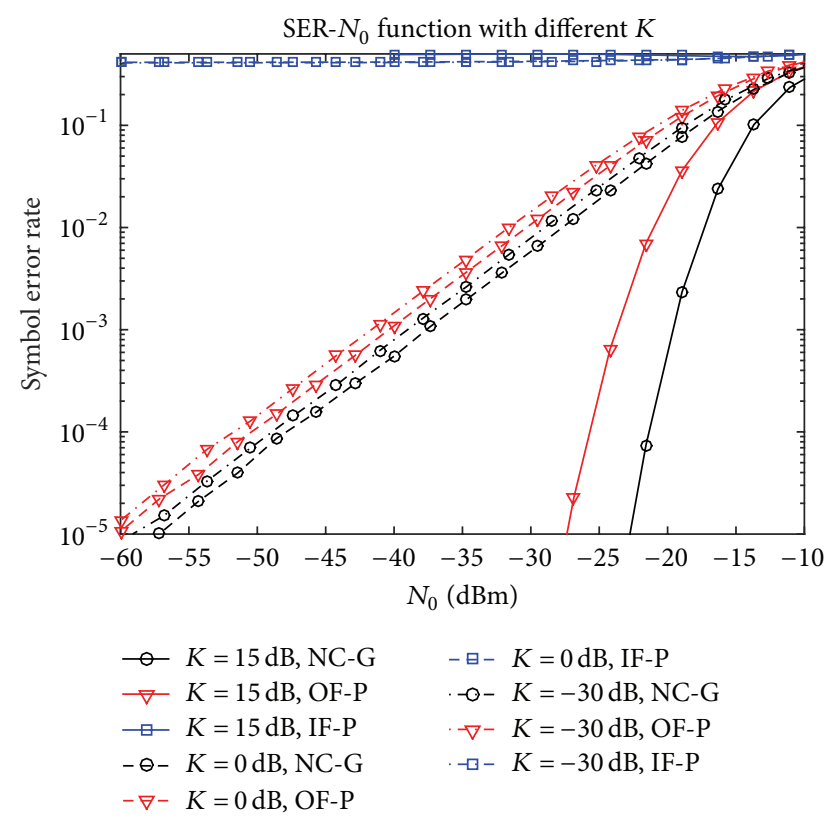

FiguRE 7: SER curves of the three schemes with different $K$.

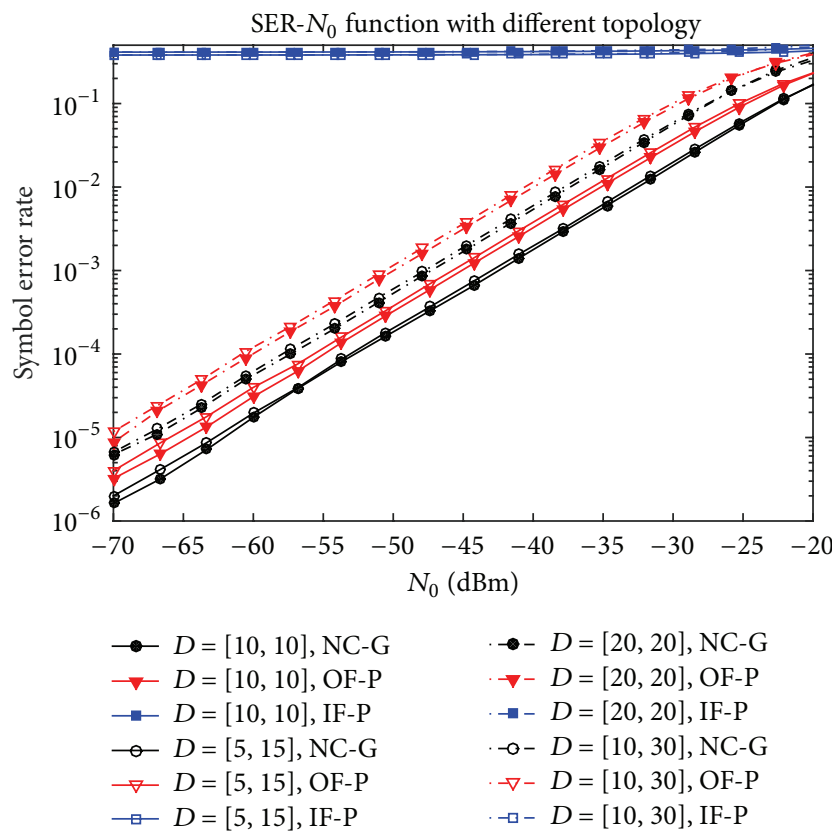

FIGURE 8: SER curves of the three schemes with different $\mathbf{d}$.

decreases, for example, changing $\mathbf{d}$ from $[10,10]$ to $[5,15]$, the SER performance remains nearly unchanged.

\section{Conclusion}

In this paper, we focus on the user-level interference under multicasting scenarios. Rather than the traditional pairwise way of D2D communications, we propose to implement the information exchanging through a groupwise way. Through introducing the idea of network coding, the proposed scheme is able to utilize the interference as valid signals to enhance the receiving performance, instead of only managing or controlling it. Both theoretical analysis and simulation results prove that the proposed scheme achieves better SINR performance and lower resources occupation than the traditional pairwise D2D transmission schemes. Besides serving as a physical transmission scheme for multicasting services in cellular networks, we expect the proposed scheme to be applicable in other network research fields for a higher network performance.

\section{Conflict of Interests}

The authors declare that there is no conflict of interests regarding the publication of this paper.

\section{Acknowledgment}

This work is supported by the National Basic Research Program of China (973 Program) 2012CB315801.

\section{References}

[1] K. Doppler, M. Rinne, C. Wijting, C. B. Ribeiro, and K. Hug, "Device-to-device communication as an underlay to LTEadvanced networks," IEEE Communications Magazine, vol. 47, no. 12, pp. 42-49, 2009.

[2] S. Hakola, C. Tao, J. Lehtoma, and T. Koskela, "Device-to-device (D2D) communication in cellular network-performance analysis of optimum and practical communication mode selection," in Proceedings of the IEEE Wireless Communications and Networking Conference (WCNC '10), pp. 1-6, April 2010.

[3] H. Min, J. Lee, S. Park, and D. Hong, "Capacity enhancement using an interference limited area for device-to-device uplink underlaying cellular networks," IEEE Transactions on Wireless Communications, vol. 10, no. 12, pp. 3995-4000, 2011.

[4] L. Lei, Z. D. Zhong, C. Lin, and X. M. Shen, "Operator controlled device-to-device communications in LTE-advanced networks," IEEE Wireless Communications, vol. 19, no. 3, pp. 96-104, 2012.

[5] 3GPP, "Technical specification group services and system aspects; feasibility study for proximity services (ProSe) (release 12)," 3GPP TR 22.803, 2012.

[6] N. Y. Lee, X. Q. Lin, J. G. Andrews, and R. W. Heath, "Power control for D2D underlaid cellular networks: modeling, algorithms, and analysis," IEEE Journal on Selected Areas in Communications, vol. 33, no. 1, pp. 1-13, 2015.

[7] G. Zhang, K. Yang, P. Liu, and J. Wei, "Power allocation for full-duplex relaying based D2D communication underlaying cellular networks," IEEE Transactions on Vehicular Technology, 2014.

[8] C. Zheng and M. Kountouris, "Distributed SIR-aware opportunistic access control for D2D underlaid cellular networks," in Proceedings of the IEEE Global Communications Conference, pp. 1540-1545, December 2014.

[9] H. ElSawy, E. Hossain, and M.-S. Alouini, "Analytical modeling of mode selection and power control for underlay D2D communication in cellular networks," IEEE Transactions on Communications, vol. 62, no. 11, pp. 4147-4161, 2014.

[10] T. D. Hoang, L. B. Le, and T. Le-Ngoc, "Joint subchannel and power allocation for D2D communications in cellular 
networks," in Proceedings of the IEEE Wireless Communications and Networking Conference (WCNC '14), pp. 1338-1343, April 2014.

[11] T. D. Hoang, L. B. Le, and L. N. Tho, "Resource allocation for D2D communications under proportional fairness," in Proceedings of the IEEE Global Communications Conference, pp. 1259-1264, December 2014.

[12] L. B. Le, "Fair resource allocation for device-to-device communications in wireless cellular networks," in Proceedings of the IEEE Global Communications Conference (GLOBECOM '12), pp. 5451-5456, December 2012.

[13] S. Mumtaz, K. M. S. Huq, A. Radwan, J. Rodriguez, and R. L. Aguiar, "Energy efficient interference-aware resource allocation in LTE-D2D communication," in Proceedings of the 1st IEEE International Conference on Communications (ICC '14), pp. 282287, June 2014.

[14] M. Shariat, A. Quddus, S. Ghorashi, and R. Tafazolli, "Scheduling as an important cross-layer operation for emerging broadband wireless systems," IEEE Communications Surveys \& Tutorials, vol. 11, no. 2, pp. 74-86, 2009.

[15] H. Min, W. Seo, J. Lee, S. Park, and D. Hong, "Reliability improvement using receive mode selection in the device-todevice uplink period underlaying cellular networks," IEEE Transactions on Wireless Communications, vol. 10, no. 2, pp. 413418, 2011.

[16] R. Tanbourgi, H. Jäkel, and F. K. Jondral, "Cooperative interference cancellation using device-to-device communications," IEEE Communications Magazine, vol. 52, no. 6, pp. 118-124, 2014.

[17] J. Jiang, M. Peng, W. Wang, and K. Zhang, "Energy efficiency optimization based on interference alignment for device-todevice MIMO downlink underlaying cellular network," in Proceedings of the IEEE Globecom Workshops, pp. 585-590, Atlanta, Ga, USA, December 2013.

[18] J. Andrusenko, R. L. Miller, J. A. Abrahamson, N. M. Merheb Emanuelli, R. S. Pattay, and R. M. Shuford, "VHF general urban path loss model for short range ground-to-ground communications," IEEE Transactions on Antennas and Propagation, vol. 56, no. 10, pp. 3302-3310, 2008. 

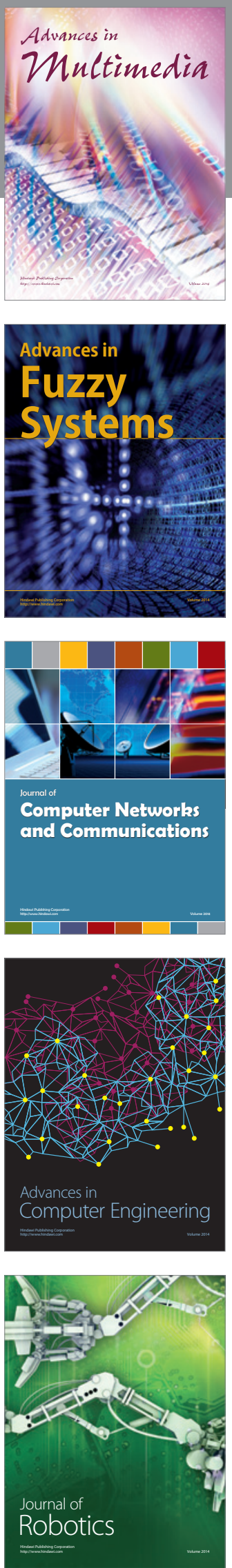

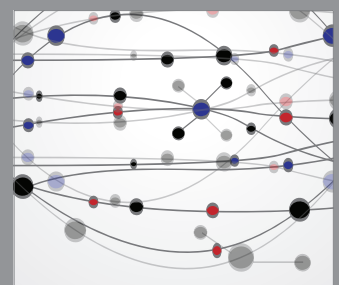

The Scientific World Journal
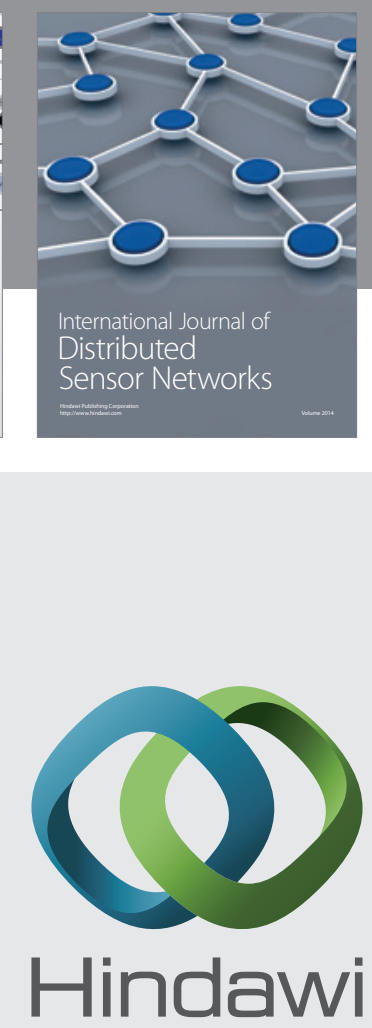

Submit your manuscripts at

http://www.hindawi.com
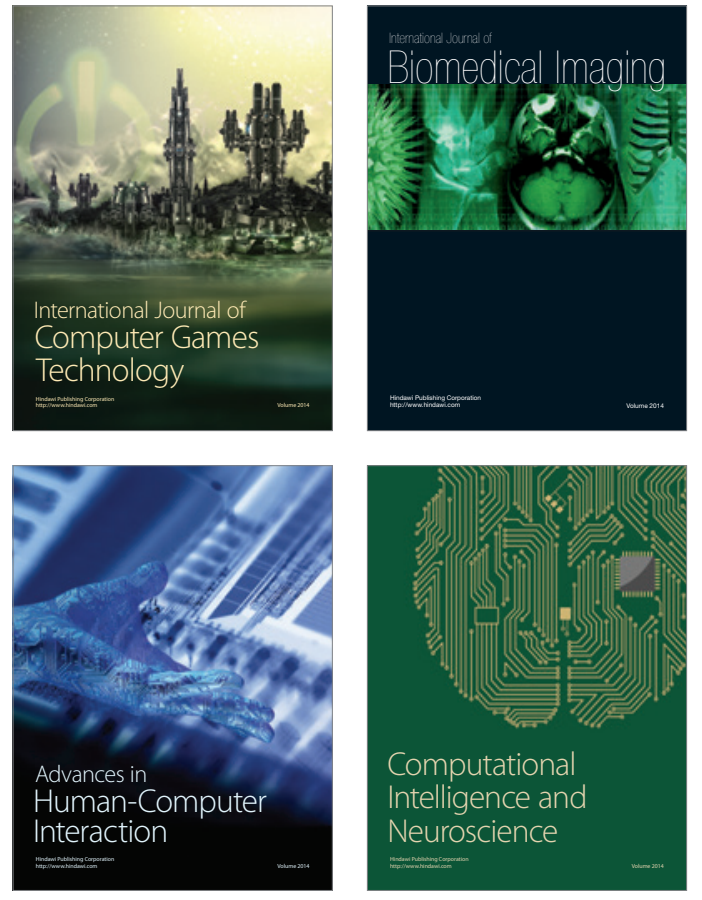
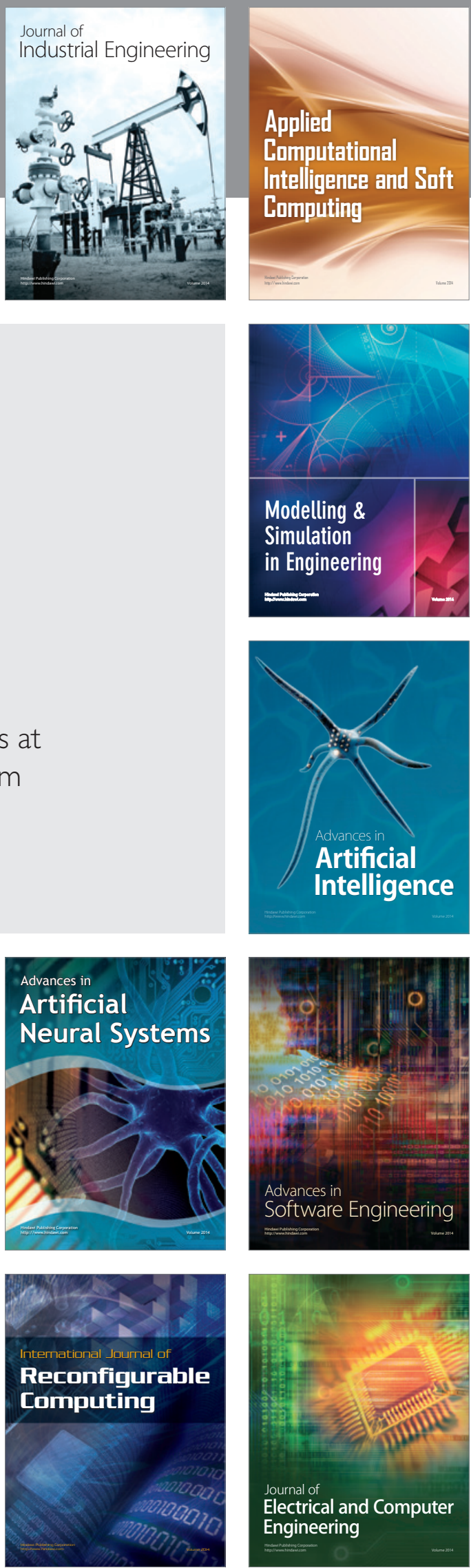